microglia when these cells are activated by neuronal insult.

In the study, 11 PGCs underwent both PK PET and ${ }^{11} \mathrm{C}$-raclopride (RAC) PET-RAC binding is a measure of striatal dopamine $D_{2}$ binding. Ten healthy controls underwent PK PET and ten underwent RAC PET. Mean striatal and cortical PK binding potential (BP) was significantly higher in PGCs than in healthy controls, whereas mean striatal RAC BP was significantly lower in PGCs, indicating loss of striatal neuronal function. Statistical parametric mapping confirmed that in PGCs PK binding was increased in several brain regions, including the striatum and cortical areas. PK and RAC BP levels were inversely correlated in individual patients. Additionally, when a patient on longterm creatine (a possible neuroprotective agent in $\mathrm{HD}$ ) was excluded, striatal PK BP—but not RAC BP-was associated with 5-year probability of disease onset. Comparisons with symptomatic patients indicated that striatal PK and RAC BP were also inversely correlated in the early to middle stages of HD.

The authors surmise that microglial activation occurs early in the pathogenesis of HD, possibly contributing to neuronal cell death and propagating disease progression. The results support future trials of agents that inhibit microglial activation in PGCs.

Original article Tai YF et al. (2007) Microglial activation in presymptomatic Huntington's disease gene carriers. Brain [doi: 10.1093/brain/awm044]

\section{Prevalence of silent brain infarction in obstructive sleep apnea}

Patients with obstructive sleep apnea (OSA) exhibit high levels of cerebrovascular mortality and morbidity. Cerebrovascular events in OSA are often preceded by silent brain infarction (SBI); however, the relationship between OSA and SBI has not been clearly defined, and the results of previous studies have been confounded by comorbidities. To address these issues, Minoguchi et al. have evaluated the prevalence of SBI, and of systemic inflammation and platelet activation, in 50 male patients with OSA and in 15 age-matched obese controls.

The investigators found that the prevalence of SBI was significantly higher in patients with moderate to severe OSA than in either patients with mild OSA or control subjects $(25.0 \%$ vs $7.7 \%$ and $6.7 \%$, respectively; $P<0.05$ for both). In addition, serum levels of markers of platelet activation (soluble CD40 ligand and soluble P-selectin) and of systemic inflammation (C-reactive protein) were elevated in patients with moderate to severe OSA compared with the other groups. Levels of these markers were also higher in patients with OSA and SBI than in those with OSA only.

The results were consistent with the idea that hypoxic stress associated with OSA mediates inflammatory responses that can lead to SBI. This proposal was further supported by the observation that 3 months of treatment with nasal continuous positive airway pressure (nCPAP) in 24 patients with moderate to severe OSA decreased levels of the above markers of systemic inflammation and platelet activation, reducing the risks for development of $\mathrm{SBI}$ and for subsequent cerebrovascular mortality and morbidity.

Original article Minoguchi K et al. (2007) Silent brain infarction and platelet activation in obstructive sleep apnea. Am J Respir Crit Care Med 175: 612-617

\section{Long-term neurological effects of enterovirus 71 infection with CNS involvement}

The long-term consequences for the CNS of enterovirus 71 (EV71) infections, which frequently present as hand, foot, and mouth disease and encephalitis, are not well understood. Chang et al. have now assessed the neurological development and cognitive function of children who survived EV71 infection with CNS involvement during the period 1998-2003 in Taiwan.

This prospective study included 142 children with clinically confirmed EV71 infection who experienced mild CNS involvement $(n=61)$, severe CNS involvement $(n=53)$, or cardiopulmonary failure following CNS infection $(n=28)$. Age at the time of disease onset was significantly lower in the most clinically severe (cardiopulmonary failure) group than in the other groups $(P<0.001)$. The results of long-term follow-up indicated that the clinical severity of CNS infection was significantly associated with the likelihood of neurological sequelae and delayed neurodevelopment; children in the most clinically severe group demonstrated high rates of neurological sequelae $(75 \%)$ and 